\title{
A classic new method to solve quartic equations
}

\author{
Amir Fathi", Nastaran Sharifan \\ Department of Electrical Engineering, Urmia branch, Islamic Azad University, Urmia, Iran \\ Department of law, Varamin-Pishva branch, Islamic Azad University, Varamin, Pishva, Iran
}

Email address:

fathi.amir@hotmail.com (A. Fathi), sharifan.nastaran@hotmail.com (N. Sharifan)

\section{To cite this article:}

Amir Fathi, Nastaran Sharifan. A Classic New Method to Solve Quartic Equations, Applied and Computational Mathematics. Vol. 2, No. 2, 2013, pp. 24-27. doi: 10.11648/j.acm.20130202.11

\begin{abstract}
Polynomials of high degrees often appear in many problems such as optimization problems. Equations of the fourth degree or so called quartics are one type of these polynomials. In this paper we give a new Classic method for solving a fourth degree polynomial equation (Quartic). We will show how the quartic formula can be presented easily at the precalculus level.
\end{abstract}

Keywords: Fourth Degree Polynomial, Quartic Equation

\section{Introduction}

Linear and quadratic equations are members of a class of equations, called polynomial equations. These equations have the general form of:

$$
a_{n} x_{n}+a_{n-1} x_{n-1}+\ldots+a_{2} x_{2}+a_{1} x+a_{0}=0
$$

In which $\mathrm{x}$ is a variable and $\mathrm{a}_{\mathrm{n}}, \mathrm{a}_{\mathrm{n}-1}, \ldots, \mathrm{a}_{2}, \mathrm{a}_{1}, \mathrm{a}_{0}$ are given constants. Also $n$ must be a positive integer and $a_{n} \neq 0$.

Lodovico Ferrari is attributed with the discovery of the solution to the quartic in 1540 , but since this solution, like all algebraic solutions of the quartic, requires the solution of a cubic to be found, it couldn't be published immediately[3]. The solution of the quartic was published together with that of the cubic by Ferrari's mentor Gerolamo Cardano in the book Ars Magna (1545).

Polynomials of high degrees often appear in problems involving optimization, and sometimes these polynomials happen to be quartics, but this is a coincidence. It is shown that any degree-n polynomial with rational (or real, or complex) coefficients has $\mathrm{n}$ complex roots. (This fact is called the fundamental theorem of algebra.) So, if we have a quadratic formula for finding both (possibly complex) roots of a quadratic (degree-2) polynomial, then it's natural to ask for a formula for all three roots of a cubic. Likewise, we would like a formula for all four roots of a quartic, and so on. It can be proved (the terms are Galois Theory and solvable groups), that there cannot exist a general formula for degree 5 and above.

In this paper we proposed a new Classic method for solving fourth degree polynomials (Quadratics) and the purpose of this paper is to show how the quartic formula can be presented easily at the precalculus level. We show how to verify that the formula is correct, and we identify when it is profitable to use it.

\section{Solving Cubic Polynomials}

If we are given a cubic equation in the form of $a y^{3}+b y^{2}+c y+d=0$ and need to solve for $\mathrm{y}$, then the first thing we do is substitute variables. Replacing eq. 1 in the given formula above,

$$
y=x-\frac{b}{3 a}
$$

We will get something in the form of:

$$
x^{3}+p x+q=0
$$

Which is the depressed cubic equation, Where

$$
\left\{\begin{array}{l}
p=-\frac{b^{2}}{3 a^{2}}+\frac{c}{a} \\
q=\frac{2 b 3}{27 a^{3}}-\frac{b c}{3 a^{2}}+\frac{d}{a} .
\end{array}\right.
$$

And replacing $x=u+v$ in the eq.2 leads to eq. 4 .

$$
u^{3}+v^{3}+3 u v(u+v)+p(u+v)+q=0
$$

This equation is changed to two equations with two unknown quantities that: 


$$
\left\{\begin{array}{l}
u^{3}+v^{3}=-q \\
u v=-\frac{p}{3} \Rightarrow u^{3} \times v^{3}=-\frac{p^{3}}{27}
\end{array}\right.
$$

By defining quadratic equation that the roots are $u^{3}$ and $v^{3}, \mathrm{u}$ and $\mathrm{v}$ can be computed.

$$
\begin{aligned}
& x^{2}-\left(u^{3}+v^{3}\right) x+u^{3} v^{3}=0 \\
& x^{2}+q x-\frac{p^{3}}{27}=0 \Rightarrow \\
& \left\{\begin{array}{l}
u=\sqrt[3]{-\frac{q}{2}+\sqrt{\frac{q^{2}}{4}+\frac{p^{3}}{27}}} \\
v=\sqrt[3]{-\frac{q}{2}-\sqrt{\frac{q^{2}}{4}+\frac{p^{3}}{27}}}
\end{array}\right. \\
& x=u+v=\sqrt[3]{-\frac{q}{2}+\sqrt{\frac{q^{2}}{4}+\frac{p^{3}}{27}}}+\sqrt[3]{-\frac{q}{2}-\sqrt{\frac{q^{2}}{4}+\frac{p^{3}}{27}}}
\end{aligned}
$$

\section{Solving Fourth Degree Polynomials (Quartics)}

The general quartic equation is:

$$
a y^{4}+b y^{3}+c y^{2}+d y+e=0 .
$$

Just as with the cubic, it behooves us to get rid of the second-highest term (in this case the cubed term) by making a substitution of variables. (To use the quadratic formula, you just plug in your coefficients). To solve Quadratic equations new method in this paper are proposed as follows:

Step1. The general quartic equation is:

$$
a y^{4}+b y^{3}+c y^{2}+d y+e=0 \text {. }
$$

It turns out that it is desirable to get rid of one of the coefficients. To accomplish this, substitute

$$
y=x-\frac{b}{4 a}
$$

Now that we've eliminated the cubic term, we have something of the form

$$
x^{4}+p x^{2}+q x+s=0
$$

Where $\mathrm{p}, \mathrm{q}$ and $\mathrm{s}$ are:

$$
\left\{\begin{array}{l}
p=-\frac{3 b^{2}}{8 a^{2}}+\frac{c}{a} \\
q=\frac{b^{3}}{8 a^{3}}-\frac{b c}{2 a^{2}}+\frac{d}{a} \\
s=-\frac{3 b^{4}}{256 a^{4}}+\frac{b^{2} c}{16 a^{3}}-\frac{b d}{4 a^{2}}+\frac{e}{a} .
\end{array}\right.
$$

$$
\left\{\begin{array}{l}
p=-\frac{3 b^{2}}{8 a^{2}}+\frac{c}{a} \\
q=\frac{b^{3}}{8 a^{3}}-\frac{b c}{2 a^{2}}+\frac{d}{a} \\
s=-\frac{3 b^{4}}{256 a^{4}}+\frac{b^{2} c}{16 a^{3}}-\frac{b d}{4 a^{2}}
\end{array}\right.
$$

Step2. With replacing $x=u+v+s$ :

$$
\begin{gathered}
(u+v+s)^{4}+p(u+v+s)^{2}+ \\
q(u+v+s)+s=0 \\
u^{4}+4 u^{3}(v+s)+6 u^{2}(v+s)^{2} \\
+4 u(v+s)^{3}+(v+s)^{4}+p\left(u^{2}+v^{2}+s^{2}\right)+ \\
2 p(u v+u s+v s)+q(u+v+s)+s=0 \\
u^{4}+v^{4}+s^{4}+4 v^{3} s+4 v s^{3}+6 v^{2} s^{2}+ \\
4 u^{3} s+6 u^{2} s^{2}+12 u v^{2} s+6 u^{2} v^{2}+4 u v^{3} \\
+12 u^{2} v s+4 u s^{3}+12 u v s^{2}+4 u^{3} v+ \\
p\left(u^{2}+v^{2}+s^{2}\right)+2 p(u v+u s+v s)+ \\
q(u+v+s)+s=0 \\
u^{4}+v^{4}+s^{4}+4 v^{3} s+4 v s^{3}+4 u^{3} v+4 u^{3} s+ \\
4 u v^{3}+4 u s^{3}+6 u^{2} v^{2}+6 u^{2} s^{2}+6 v^{2} s^{2}+ \\
8 u v s^{2}+4 u v s^{2}+8 u v^{2} s+4 u v^{2} s+8 u^{2} v s+ \\
4 u^{2} v s+p\left(u^{2}+v^{2}+s^{2}\right)+2 p(u v+u s+v s)+ \\
q(u+v+s)+s=0
\end{gathered}
$$

Step3. This equation is changed to three equations with three unknown quantities, only under this condition that the previous equation is satisfied.

$$
8 u v s^{2}+8 u v^{2} s+8 u^{2} v s+q(u+v+s)=0
$$

Firs equation is defined:

$$
u v s=-\frac{q}{8}
$$

Residue of the equation (14) is:

$$
\begin{aligned}
& u^{4}+v^{4}+s^{4}+4\left(u^{2}+v^{2}+s^{2}\right)(u v+u s+v s)+ \\
& p\left(u^{2}+v^{2}+s^{2}\right)+2 p(u v+u s+v s)+s+ \\
& 6\left(u^{2} v^{2}+u^{2} s^{2}+v^{2} s^{2}\right)=0
\end{aligned}
$$

Second equation is defined as below:

$$
4\left(u^{2}+v^{2}+s^{2}\right)(u v+u s+v s)+2 p(u v+u s+v s)=0
$$




$$
\begin{gathered}
(u v+u s+v s)\left(4\left(u^{2}+v^{2}+s^{2}\right)+2 p\right)=0 \rightarrow \\
(u v+u s+v s) \neq 0 \Rightarrow \\
4\left(u^{2}+v^{2}+s^{2}\right)+2 p=0 \rightarrow \\
u^{2}+v^{2}+s^{2}=-p / 2
\end{gathered}
$$

Residue of the equation (17) is:

$$
\begin{aligned}
& \left(u^{4}+v^{4}+s^{4}\right)-\frac{p^{2}}{2}+s+ \\
& 6\left(u^{2} v^{2}+u^{2} s^{2}+v^{2} s^{2}\right)=0
\end{aligned}
$$

And:

$$
\begin{gathered}
u^{2}+v^{2}+s^{2}=-\frac{p}{2} \stackrel{\text { by squaring }}{\longrightarrow} \\
\left(u^{2}+v^{2}+s^{2}\right)^{2}=\left(-\frac{p}{2}\right)^{2} \\
u^{4}+v^{4}+s^{4}+ \\
2\left(u^{2} v^{2}+u^{2} s^{2}+v^{2} s^{2}\right)=\frac{p^{2}}{4} \\
-\stackrel{i f}{\longrightarrow} u^{4}+v^{4}+s^{4}=A \\
u^{2} v^{2}+u^{2} s^{2}+v^{2} s^{2}=B \Rightarrow \\
A+2 B=\frac{p^{2}}{4}
\end{gathered}
$$

It is concluded from equation (19):

$$
A+6 B=\frac{p^{2}}{2}-s
$$

With solving the two equations with two unknown quantities that named (20) and (21) it is obtained:

$$
\begin{aligned}
B & =\frac{p^{2}}{16}-\frac{s}{4} \Rightarrow \\
B & =u^{2} v^{2}+u^{2} s^{2}+v^{2} s^{2}=\frac{p^{2}}{16}-\frac{s}{4} \\
A & =\frac{p^{2}}{4}-\frac{p^{2}}{8}+\frac{s}{2}=\frac{p^{2}}{8}+\frac{s}{2} \\
& \Rightarrow A=u^{4}+v^{4}+s^{4}=\frac{p^{2}}{8}+\frac{s}{2}
\end{aligned}
$$

By squaring the expression (22) we will have:

$$
B^{2}=u^{4} v^{4}+u^{4} s^{4}+v^{4} s^{4}+2 u^{2} v^{2} s^{2}\left(u^{2}+v^{2}+s^{2}\right)
$$

$$
\begin{array}{r}
B^{2}=u^{4} v^{4}+u^{4} s^{4}+v^{4} s^{4}+\frac{2 q}{64} \times\left(-\frac{p}{2}\right) \\
u^{4} v^{4}+u^{4} s^{4}+v^{4} s^{4}=B^{2}+\frac{q^{2} p}{64}
\end{array}
$$

From equation (16):

$$
u v s=-\frac{q}{8} \Rightarrow u^{4} v^{4} s^{4}=\left(\frac{q}{8}\right)^{4}
$$

Using expressions (23), (24), and (25) we define a cubic equation such that roots are $\mathrm{u}^{4}, \mathrm{v}^{4}$ and $\mathrm{s}^{4}$ and also three previous expressions are satisfied. Cubic equation is defined as below:

$$
\begin{aligned}
& N^{3}-\left(u^{4}+v^{4}+s^{4}\right) N^{2}+\left(u^{4} v^{4}+u^{4} s^{4}+v^{4} s^{4}\right) N- \\
& \left(u^{4} v^{4} s^{4}\right)=0 \\
& \Rightarrow N^{3}-\left(\frac{p^{2}}{8}+\frac{s}{2}\right) N^{2}+\left[\left(\frac{p^{2}}{16}-\frac{s}{4}\right)^{2}+\right. \\
& \left.\frac{q^{2} p}{64}\right] N-\left(\frac{q}{8}\right)^{4}=0
\end{aligned}
$$

Naming the equation roots $N_{1}, N_{2}, N_{3}\left(u^{4}, v^{4}, s^{4}\right)$ respectively, this expression is concluded:

$$
x=\sqrt[4]{N_{1}}+\sqrt[4]{N_{2}}+\sqrt[4]{N_{3}}
$$

\section{Examples}

Find four roots of $x^{4}+8 x+4=0$.

Solution:

$$
\begin{gathered}
x^{4}+8 x+4=0 \Rightarrow q=8, s=4, p=0 \\
\Rightarrow A=2, B=-1 \\
N^{3}-2 N^{2}+N-1=0 \\
N_{1}=1.7549 \angle 0 \\
N_{2}=0.1226+0.7449 i=0.7549 \angle 80.65 \\
N_{3}=0.1226-0.7449 i=0.7549 \angle-80.65
\end{gathered}
$$
s4):

With extracting the square root of ${ }^{L \mathbf{v}_{1}, L \mathbf{v}_{2}, L \mathbf{v}_{3}}$ (u4, v4,

$$
\sqrt{N_{1}}=1.3247 \angle 0,1.3247 \angle 180 \longrightarrow 1.3247,-1.3247
$$

$$
\begin{aligned}
& \sqrt{N_{2}}=0.8688 \angle 40.32,0.8688 \angle 220.3 \longrightarrow \\
& 0.6624+0.5621 i,-0.6224-0.5619 i
\end{aligned}
$$




$$
\begin{aligned}
& \sqrt{N_{3}}=0.8688 \angle-40.32,0.8688 \angle 139.67 \longrightarrow \\
& 0.6624-0.5622 i,-0.6624+0.5622 i
\end{aligned}
$$

The roots which satisfy equation (18) are selected:

$$
\begin{gathered}
\sqrt{N_{1}}=1.3247 \\
\sqrt{N_{2}}=-0.6224-0.5619 i \\
\sqrt{N_{3}}=-0.6624+0.5622 \\
\sqrt[4]{N_{1}}=0.3210-0.8749,-0.3210+0.8749 i \\
\sqrt[4]{N_{2}=}=0.3210+0.8749 i,-0.3210-0.8749 i \\
\sqrt[4]{N_{3}}=1.15095,-1.15095
\end{gathered}
$$

The answers which satisfy equation (16) are selected:

$$
\begin{gathered}
x=u+v+s \Rightarrow \\
x_{1}=-1.7929 \\
x_{2}=-0.5089 \\
x_{3}=1.1510+1.75 i \\
x_{4}=1.1510-1.75 i
\end{gathered}
$$

By using the second method we verify the following example.

\section{Conclusions}

The most important point in all of the methods for solving a quartic equation is the complexity of these solutions.

To prove the efficiency and simplicity of the proposed method an example quartic is given in the fourth section of the paper and it is solved with the proposed initiated method.

\section{References}

[1] "A Simple Method to Solve Quartic Equations" Amir Fathi, Pooya Mobadersany, Rahim Fathi, Australian Journal of
Basic and Applied Sciences, 6(6): 331-336, 2012, ISSN 1991-8178.

[2] Cardano, Girolamo, (translated by T. Richard Witmer), Ars Magna or the Rules of Algebra, Dover, New York, NY, 1993.

[3] Faucette, W. M. "A Geometric Interpretation of the Solution of the General Quartic Polynomial." Amer. Math. Monthly 103, 51-57, 1996.

[4] Gellert, W.; Gottwald, S.; Hellwich, M.; Kästner, H.; and Künstner, H. (Eds.). VNR Concise Encyclopedia of Mathematics, 2nd ed. New York: Van Nostrand Reinhold, 1989.

[5] Hazewinkel, M. (Managing Ed.). Encyclopaedia of Mathematics: An Updated and Annotated Translation of the Soviet "Mathematical Encyclopaedia." Dordrecht, Netherlands: Reidel, 1988.

[6] MathPages. "Reducing Quartics to Cubics." http://www.mathpages.com/home/kmath296.htm.

[7] Smith, D. E. A Source Book in Mathematics. New York: Dover, 1994.

[8] van der Waerden, B. L. $\$ 64$ in Algebra, Vol. 1. New York: Springer-Verlag, 1993.

[9] Beyer, W. H. CRC Standard Mathematical Tables, 28th ed. Boca Raton, FL: CRC Press, p. 12, 1987a.

[10] Beyer, W. H. Handbook of Mathematical Sciences, 6th ed. Boca Raton, FL: CRC Press, 1987b.

[11] Birkhoff, G. and Mac Lane, S. A Survey of Modern Algebra, 5th ed. New York: Macmillan, pp. 107-108, 1996.

[12] Borwein, P. and Erdélyi, T. "Quartic Equations." §1.1.E.1e in Polynomials and Polynomial Inequalities. New York: Springer-Verlag, p. 4, 1995.

[13] Boyer, C. B. and Merzbach, U. C. A History of Mathematics, 2nd ed. New York: Wiley, pp. 286-287, 1991.

[14] I. Stewart, "Galois theory," ed: Chapman \& Hall/CRC Mathematics, 2004.

[15] J. J. O'Connor and E. F. Robertson, "Lodovico Ferrari," in The MacTutor History of Mathematics archive, ed. School of Mathematics and Statistics, University of St Andrews Scotland.

[16] AN EASY LOOK AT THE CUBIC FORMULA .Thomas J. Osler.Mathematics Department, Rowan University, Glassboro NJ 08028. 